\title{
EXAMINATION OF DIETARY PATTERNS AND FODMAP INTAKE IN PATIENTS WITH IRRITABLE BOWEL SYNDROME
}

\author{
A Thesis \\ Presented to Graduate with Distinction from the \\ School of Health and Rehabilitation Sciences
}

The Ohio State University

2019

Thesis Examination Committee:

Dr. Kristen Roberts, Advisor

Dr. Marcia Nahikian-Nelms 


\section{Background and Problem Statement}

Irritable bowel syndrome (IBS) is a group of disorders classified as functional gastrointestinal disorders. IBS includes three subtypes: IBS-D (diarrhea predominant), IBS-C (constipation predominant), and IBS-M (mixed diarrhea and constipation). ${ }^{1}$ The specific etiology of IBS is unknown, but potential risk factors include genetic predisposition, altered immune response, elevated inflammatory response, small intestinal bacterial overgrowth, and increased sensitivity of the enteric nervous system. ${ }^{2}$ In North America, approximately $10 \%$ of individuals have symptoms associated with IBS. $^{2}$ It is also a worldwide complaint with an estimated global prevalence of $11.2 \%$ and the rate has remained stable in the last 30 years. ${ }^{3}$

Currently, there is no diagnostic test and effective treatment for IBS. Physicians make a diagnosis after excluding all other possible organic causes of symptoms. The Rome IV Criteria is used to assist providers in diagnosing IBS which uses classic IBS symptoms such as abdominal pain, altered bowel habits, and abdominal distension/bloating for diagnosis. ${ }^{4}$ Other gastrointestinal (GI) disorders, such as celiac disease and lactose maldigestion, are frequently associated with IBS. The presently ineffective medical treatment for IBS increases the frequency of doctor visits, diagnostic tests, and hospitalizations for patients with IBS compared to those without IBS which ultimately causes financial stress on patients with IBS.

Recent studies have indicated that fermentable oligo-, di-, and monosaccharides, and polyols (FODMAPs) are a significant factor in exacerbating IBS symptoms. ${ }^{4}$ The poorly absorbed carbohydrates become a food source for bacterial fermentation in the small intestine and colon. The bacterial fermentation produces gas which results in abdominal distension and abdominal discomfort. ${ }^{4}$ Additionally, some FODMAPs are osmotically active and can pull fluid 
into the GI tract leading to diarrhea in some. ${ }^{4}$ Understanding and assessing the dietary patterns in IBS may elucidate the role of diet in managing and controlling symptoms.

The treatment of IBS focuses on improving symptoms and quality of life. A diet low in FODMAPs has been proposed as an efficacious dietary pattern that reduces symptoms of IBS. In a study by xx et al assessing dietary interventions in IBS, those following a low FODMAP diet reported relief of GI symptoms. ${ }^{13}$ In order to understand this phenomenon, a basic estimation of the FODMAP content of the diet and the corresponding food sources is of interest. This information may, in turn, be used to generate appropriate nutrition intervention for IBS that could target specific FODMAPs in the diet. Food frequency questionnaires (FFQs) are a dietary assessment tool used to estimate dietary consumption over a period of 90 days. ${ }^{5}$ From this data, certain FODMAPs can be estimated, such as lactose and fructose. Gluten is a protein found in wheat, rye and barley. Unfortunately, it is not included in the analysis of FFQ data. The gluten content of the diet could be used as a surrogate marker for wheat, rye and barley intake which are all restricted on a low FODMAP diet as well due to their fructan content. Unfortunately, fructan content is unavailable with most FFQ data sets. Although not all FODMAPs are available through FFQ data, utilizing these estimates may allow us to generally compare the estimated, average consumption of FODMAPs between people with and without IBS. This leads to hypothesis generation about the dietary patterns of those with IBS as it relates to FODMAP content. Additionally, the Registered Dietitian Nutritionist (RDN) can utilize this data to better understand the dietary patterns as it relates to FODMAP intake to adjust and modify the nutrition interventions in this cohort. 


\section{Literature Review}

\section{Prevalence}

IBS is a chronic GI functional disorder that affects $9 \%-23 \%$ of the population across the world. ${ }^{6}$ According to the American College of Gastroenterology, 10\%-15\% percent of adult population in the United States suffers from IBS symptoms, but only 5\%-7\% percent of them have been diagnosed. ${ }^{7}$ In the United States, Canada, and Israel, women have about 1.5 to 2 times rate diagnosed for IBS symptoms than men. ${ }^{8}$ Given this high prevalence worldwide, it is important to understand the pathophysiology of IBS to develop effective treatments for reduction of gastrointestinal symptoms.

\section{Etiology and Pathophysiology}

The etiology and pathophysiology of IBS are incompletely understood but several factors such as genetic/social factors, intestinal microbiota, low-grade chronic intestinal inflammation, abnormal GI endocrine cells, and dietary intake appear to be associated with the pathophysiology of IBS. ${ }^{4}$ There is no convincing evidence to support that food allergy is a causative factor in the pathophysiology of IBS. ${ }^{4,8}$ However, many patients have reported that their symptoms worsen by eating certain food items, some being those foods rich in FODMAPs such as wheat, rye, asparagus, sprouts, apples, figs and legumes. ${ }^{8}$ FODMAPs contained in these foods include fructose, lactose, fructans, galactans, glucto-oligosaccharides and polyols (sorbitol, mannitol, maltitol, xylitol, erythritol, polydextrose, and isomalt). The indigestible and poorly absorbed short-chain carbohydrates in FODMAPs have shown osmotic activity in the intestinal luminal

and colon leading to the hypothesized change in stool consistency and frequency. ${ }^{10}$ Additionally, FODMAPs provide food sources for bacterial fermentation causing gas production, bowel 
distension, bloating, cramping, and diarrhea ${ }^{10}$ Fructose is commonly found in fruits (ie., apples and watermelon), honey, and high-fructose corn syrup. Free fructose does not require additional digestion since it is absorbed by the small intestinal epithelium. Fructose in the GI tract can lead to a concentration gradient where the concentration of fructose is higher in the lumen than in the intestinal epithelial cell allowing fructose to migrate to the terminal ileum in the GI tract leading to malabsorption in some with IBS and subsequent fermentation. ${ }^{8}$ Lactose naturally is found in mammalian milk. In most cases, lactose intolerance is a result of inadequate lactase secreted at the brush border. ${ }^{10}$ Fructans and galactans are oligosaccharides mainly found in wheat and legumes. The human intestinal tract does not secrete enzymes to digest these nutrients and break them down for absorption into the epithelial cells. Therefore, the unabsorbed fructans and galactans result in fermentation and gas formation. Lastly, polyols are sugar alcohols utilized mainly as sugar substitutes. Only around one-third intake of polyols is actually absorbed through passive diffusion in the small intestine epithelial. ${ }^{10}$ The speed of absorption of polyols is slow and is related to molecular size, variation of pore size of the small intestine, and pore size affected by mucosal disease. ${ }^{10}$ The smaller molecular size and/or larger pore size along the small intestine increases polyol absorption. ${ }^{10}$ Then, some diseases such as celiac disease can decrease the pore size leading to absorption of polyols. ${ }^{10}$ It is clear that these poorly absorbed carbohydrates could be partially related to the symptoms seen in IBS. Therefore, understanding the absolute delivery and sources of FODMAPs in the diet can play an important role in nutrition therapy to improve IBS symptoms.

Table 1. Summary of FODMAPs

\begin{tabular}{|l|l|l|}
\hline \multicolumn{1}{|c|}{ FODMAP } & \multicolumn{1}{|c|}{$\begin{array}{c}\text { Associated with } \\
\text { Pathophysiology }\end{array}$} & \multicolumn{1}{c|}{ Food Sources } \\
\hline Fructose & $\begin{array}{l}\text { Absorbed by the small bowl } \\
\rightarrow \text { a concentration gradient }\end{array}$ & $\begin{array}{l}\text { Fruit, honey, high-fructose } \\
\text { corn syrup }\end{array}$ \\
\hline
\end{tabular}




\begin{tabular}{|l|l|l|}
\hline Lactose & Inadequate lactase secretion & Milk, cheese, yogurt \\
\hline Fructans/ Galactans & $\begin{array}{l}\text { No enzymes to break down } \\
\rightarrow \text { fermentation and gas } \\
\text { formation }\end{array}$ & Wheat, legumes \\
\hline Polyols & $\begin{array}{l}\text { Slowly absorbed by passive } \\
\text { diffusion }\end{array}$ & $\begin{array}{l}\text { Sugar alcohols, sugar } \\
\text { substitutes }\end{array}$ \\
\hline
\end{tabular}

\section{Diagnosis}

Currently, there is no golden standard diagnostic criteria for IBS because the unknown etiology and pathophysiology of this syndrome. Symptoms usually are not consistent in patients with IBS, so criteria have been developed to identify a combination of symptoms to increase the accurate of diagnosing IBS. The first published diagnostic criteria are Manning in the $1979 .{ }^{6}$ Over the last several decades, this criteria has developed into the current Rome IV criteria. The Rome IV criteria asserts that patients must have recurrent abdominal pain on average at least 1 day per week during the past 3 months. ${ }^{9}$ In addition, the patient must have at least two following features: related to defecation, onset associated with a change in stool frequency, or onset associated with a change in stool consistency. ${ }^{9}$ In 2009, the American College of Gastroenterology (ACG) publishing a position paper stating that people are diagnosed with IBS if abdominal pain or discomfort occurring in association with altered bowel habits persists over a period of at least 3 months. ${ }^{6}$

\section{Medical Management}

IBS is a symptom-based disorder resulting from diverse pathologies as mentioned, so medical management is targeted to treat GI related symptoms such as diarrhea, constipation, pain, bloating, and cramping. For patients with IBS-D, the first-line agents are antidiarrheal medications, such as loperamide, to inhibit peristalsis, prolong gut transit, and reduce fecal volume. ${ }^{8}$ If patients with IBS-D do not respond to antidiarrheal medications, serotonin targeted 
agents, such as Alosetron and Ondansetron which are 5-HT3 receptor antagonists, are used to improve gastrointestinal motility and visceral sensation. ${ }^{8}$ Then, the third type of medication for IBS-D are antispasmodics which are drugs with anticholinergic or calcium channel blocking properties. They help gut smooth muscle to relax. ${ }^{8}$ For patients with IBS-C, the medical management includes fiber supplements, laxative agents, and prosecretory agents. ${ }^{8}$ Additionally, soluble fiber has shown greater efficacy to treat IBS-C symptoms rather than insoluble fiber. ${ }^{8}$ Osmotic laxatives such as polyethylene glycol is a medication that pulls water into the colon to soften stool to improve constipation. ${ }^{8}$ Lastly, prosecretory agents stimulate intestinal fluid secretion to solve chronic constipation. Regardless of the subtyping, probiotics, antibiotics, and antidepressants are commonly prescribed to patients with IBS. Probiotics are live bacteria that provide general health benefits to the host. ${ }^{8}$ Rifaximin is an antibiotic has been evaluated in IBS for treatment of small intestinal bacterial overgrowth. Some efficacy has been seen with these studies which researchers hypothesize is related to the bacterial load present in those with IBS. ${ }^{8}$ Antidepressants such as tricyclic antidepressants, are utilized frequently in those with IBS due to the effect of selective serotonin-reuptake inhibitors on mood and gastrointestinal motility. Despite the array of medical management available, data continues to support the efficacy of symptom improvement with the low FODMAP diet compared to medications.

\section{Nutritional Management}

Recently, dietary interventions to control and treat IBS symptoms have received more attention because people with IBS report that they are able to identify some foods triggering their symptoms. ${ }^{11}$ Many diets have been studied in those with IBS including a gluten-free diet, strict elimination diets, low lactose diets and others, but poor efficacy has abandoned their use worldwide until the release of the low FODMAP diet. The low-FODMAP diet is commonly 
employed by patients with IBS. The low-FODMAP diet is designed for short-term use and the purposed is to identify if symptoms are related to the poor digestion and absorption of shortchained carbohydrates. The low-FODMAP diet intervention includes of 3 distinct phases: the restriction or elimination phase, the reintroduction or rechallenge phase, and the maintenance or personalized phase. ${ }^{12}$ During the first phase, patients remove all FODMAPs from their diet for up to 8 weeks. ${ }^{12}$ In those reporting improved symptoms, they move to the reintroduction phase to identify which foods were culprits. During the third and final phase, people continue the consumption of FODMAPs that were well-tolerated and continue eliminating trigger foods. ${ }^{12}$

The impact of this dietary approach has now been studied by many researchers.

Consistently, approximately $75 \%$ of patients with IBS experience symptom improvement when abdominal pain and bloating were the predominant symptoms. ${ }^{12}$ In those with constipation or diarrhea just over $50 \%$ respond to a low-FODMAP diet. ${ }^{12}$ Although questions still remain unanswered related to dietary compliance and the potential long term effects, it is still commonplace to start with this dietary intervention in those with a diagnosis of IBS.

\section{Assessment of Dietary Patterns}

Fructose, lactose, and polyols are main components of FODMAPs which are important contributors of IBS. There are several tools to assess dietary patterns, yet no tool has been optimized to assess the total FODMAP content of this diet that is available in public. The USDA food database provides an accessible tool that can quantify a few FODMAPs in the diet (such as fructose and lactose). Given that FFQs utilize this database, using an FFQ could be considered for dietary analysis when attempting to quantify dietary FODMAP patterns. Strengths of an FFQ includes the ability to capture the dietary pattern over a three month period, ability to capture episodically consumed foods, and electronic data can be quickly collected and analyzed. ${ }^{5}$ FFQs 
are traditionally utilized in the research setting because it is useful in large populations with potential low cost and low participant burden. However, the weakness of FFQ is that it not suitable for cross-cultural comparisons since it is based on American eating style and it requires good memory, literacy, and numerical sills. ${ }^{5}$ VioScreen ${ }^{\circledR}$ is a pictorial, web-based application of an FFQ which offers improved security and privacy of participant information. ${ }^{5}$ It reduces time and is validated for high accuracy. ${ }^{5}$ The pictures in the VioScreen ${ }^{\circledR}$ allow a more accurate representation of portion sizes ultimately improving the accuracy of the dietary analysis.

\section{Objectives}

The goal of this study is to evaluate the dietary patterns of patients with and without IBS to determine the average intake of fructose, lactose and polyols. With this data, we can address if those with IBS are consuming more or less FODMAPs in the diet to improve the RDNs understanding of usual dietary intake of those with IBS. With this information, the RDN can optimize dietary education by understanding food sources of FODMAPs typically consumed in those with IBS. The following research questions will be explored:

- What is the average consumption of fructose, lactose, and polyols in the patients with and without IBS as measured by food frequency questionnaire (FFQs)?

- Do patients with IBS consumed less or more fructose, lactose, and polyols compared to patients without IBS as measured by FFQ?

\section{Method}

Subjects

FFQs are offered to all patients in the outpatient Gastroenterology, Hepatology and Nutrition (GHN) clinic at The Ohio State University Wexner Medical Center (OSUWMC). Participants 
completing this FFQ between April 2018 to November 2019 were used to estimate average consumption of fructose, lactose, and polyols. Average dietary consumption of fructose, lactose, and polyols was compared between participants with IBS and participants without IBS. Approval from the Institutional Review Board (IRB) was received prior to data collection (2016H0320).

\section{Data Collection}

Patients within the GHN outpatient clinic at OSUWMC were all provided with a handout indicating their access code to the on-line FFQ. Patients completed VioScreen ${ }^{\mathrm{TM}} \mathrm{FFQ}$ on their computer or other electronic devices. Charts were reviewed to confirm all GI diagnoses. All dietary information, anthropometric data, exercise patterns, multivitamin use and age were selfreported through the FFQ platform. Any participant that was not seen in a GHN clinic was excluded from analysis.

Dietary Analysis

Data collected from VioScreen ${ }^{\mathrm{TM}}$ was used to evaluate the dietary patterns for patients with or without IBS and to measure average consumption of fructose, lactose, and polyols. For the purpose of this study, only those with an intake $>500 \mathrm{kcal} / \mathrm{day}$ will be included. To estimate total polyols in the diet, a sum of erythritol, inositol, isomalt, lactitol, maltitol, mannitol, pinitol, sorbitol, and xylitol were used to determine total polyol consumption in the diet as these are the polyols available in the FFQ for analysis. Each category of FODMAPs were totaled and averaged to estimate the contribution of these FODMAPS within the diet.

\section{Statistics}

Descriptive statistics were used to describe the dietary patterns in patients with IBS and compare it with patients without IBS. Average daily fructose, lactose, and polyols consumption, and additional continuous variables were represented as means \pm standard deviations. 


\section{Results}

\section{Demographics and Anthropometrics}

A total of 145 participants with GI diseases completed the Vioscreen ${ }^{\mathrm{TM}}$ within the defined time period. Five participants were excluded. One participant did not have a name that matched the date of birth in the electronic medical record, three participants did not have an electronic medical record at OSUWMC, and one participant had a duplicated entry. Therefore, the final sample size was 140 records. Of these, $82.9 \%$ participants were not diagnosed with IBS $(n=116)$ and $17.1 \%$ participants were diagnosed with IBS $(n=24)($ see Table 1). For all

participants $(\mathrm{N}=140)$, average age was $43.0 \pm 15.5$ years with an average weight of $80.2 \pm 22.9$ kg. Participants tended to be overweight with a BMI of $28.2 \pm 7.4 \mathrm{~kg} / \mathrm{m}^{2}$ (see Table 1). The results indicate majority of participants $(\mathrm{N}=140)$ has low activity level $(\mathrm{n}=64,45.7 \%$, see Table 1). Only $54(38.6 \%)$ participants report that they take multivitamin and $86(61.4 \%)$ participants deny for it (see Table 1).

Table 1. Demographics Characteristics 


\begin{tabular}{|c|c|c|}
\hline \multicolumn{2}{|c|}{ Characteristic } & $N=140$ \\
\hline IBS & $\begin{array}{l}\text { Yes, n (\%) } \\
\text { No, } \mathrm{n}(\%)\end{array}$ & $\begin{array}{l}24(17.1 \%) \\
116(82.9 \%)\end{array}$ \\
\hline Anthropometrics & $\begin{array}{l}\text { Age in years, } \\
\text { median (range); } \\
\text { Weight in } \mathrm{kg}, \\
\text { median (range); } \\
\mathrm{BMl} \text { in } \mathrm{kg} / \mathrm{m}^{2} \text {, } \\
\text { median (range) }\end{array}$ & $\begin{array}{l}43.0(27.5-58.5) \\
80.2(57.3-103.1) \\
28.2(20.8-35.6)\end{array}$ \\
\hline Activity Level & $\begin{array}{l}\text { Sedentary, } \mathrm{n}(\%) \\
\text { Low active, } \mathrm{n}(\%) \\
\text { Active, } \mathrm{n}(\%) \\
\text { Very Active, } \mathrm{n}(\%)\end{array}$ & $\begin{array}{l}16(11.4 \%) \\
64(45.7 \%) \\
48(34.3 \%) \\
12(8.6 \%)\end{array}$ \\
\hline $\begin{array}{l}\text { Multivitamin } \\
\text { Intake }\end{array}$ & $\begin{array}{l}\text { Yes, } n(\%) \\
\text { No, } n(\%)\end{array}$ & $\begin{array}{l}54(38.6 \%) \\
86(61.4 \%)\end{array}$ \\
\hline
\end{tabular}

Dietary intake including kilocalories, macronutrient, and fiber from the Vioscreen ${ }^{\mathrm{TM}} \mathrm{FFQ}$ were analyzed (see Table 2). Results indicate total participants $(\mathrm{N}=140)$ consumed $1824.7 \pm 1046.8$ kcals/day (see Table 2). Those with IBS $(\mathrm{n}=24)$ consumed similar kilocalories compared to that of participants without IBS $(\mathrm{n}=116)(2065.6 \pm 1538.1$ vs $1774.9 \pm 914.7 \mathrm{kcal}$, respectively; see Table 2). The results show that the contribution of kilocalories coming from macronutrients by diagnosis of IBS are identify in two study groups. (see Figure 1).

Table 2. Dietary Intake as Measured by Vioscreen ${ }^{\mathrm{TM}}$ Report

\begin{tabular}{|l|l|l|l|}
\hline & Total sample $(\mathbf{N}=\mathbf{1 4 0})$ & No IBS $(\mathbf{n}=\mathbf{1 1 6})$ & IBS $(\mathbf{n}=\mathbf{2 4})$ \\
\hline & Mean \pm SD & Mean \pm SD & Mean \pm SD \\
\hline
\end{tabular}




\begin{tabular}{|l|l|l|l|}
\hline Kilocalorie (kcal/d) & $1824.7 \pm 1046.8$ & $1774.9 \pm 914.7$ & $2065.6 \pm 1538.1$ \\
\hline Carbohydrate $(\mathrm{g} / \mathrm{d})$ & $216.2 \pm 147.3$ & $208.2 \pm 131.8$ & $254.7 \pm 205.8$ \\
\hline Fat $(\mathrm{g} / \mathrm{d})$ & $75.5 \pm 46.2$ & $73.9 \pm 41.7$ & $83.1 \pm 64.0$ \\
\hline Protein $(\mathrm{g} / \mathrm{d})$ & $73.6 \pm 41.6$ & $72.5 \pm 37.2$ & $79.0 \pm 59.1$ \\
\hline Fiber $(\mathrm{g} / \mathrm{d})$ & $19.1 \pm 11.3$ & $18.6 \pm 10.3$ & $21.3 \pm 15.4$ \\
\hline
\end{tabular}

Figure 1. Comparison of Macronutrients Intake Between Participants with IBS and without IBS

$60 \%$

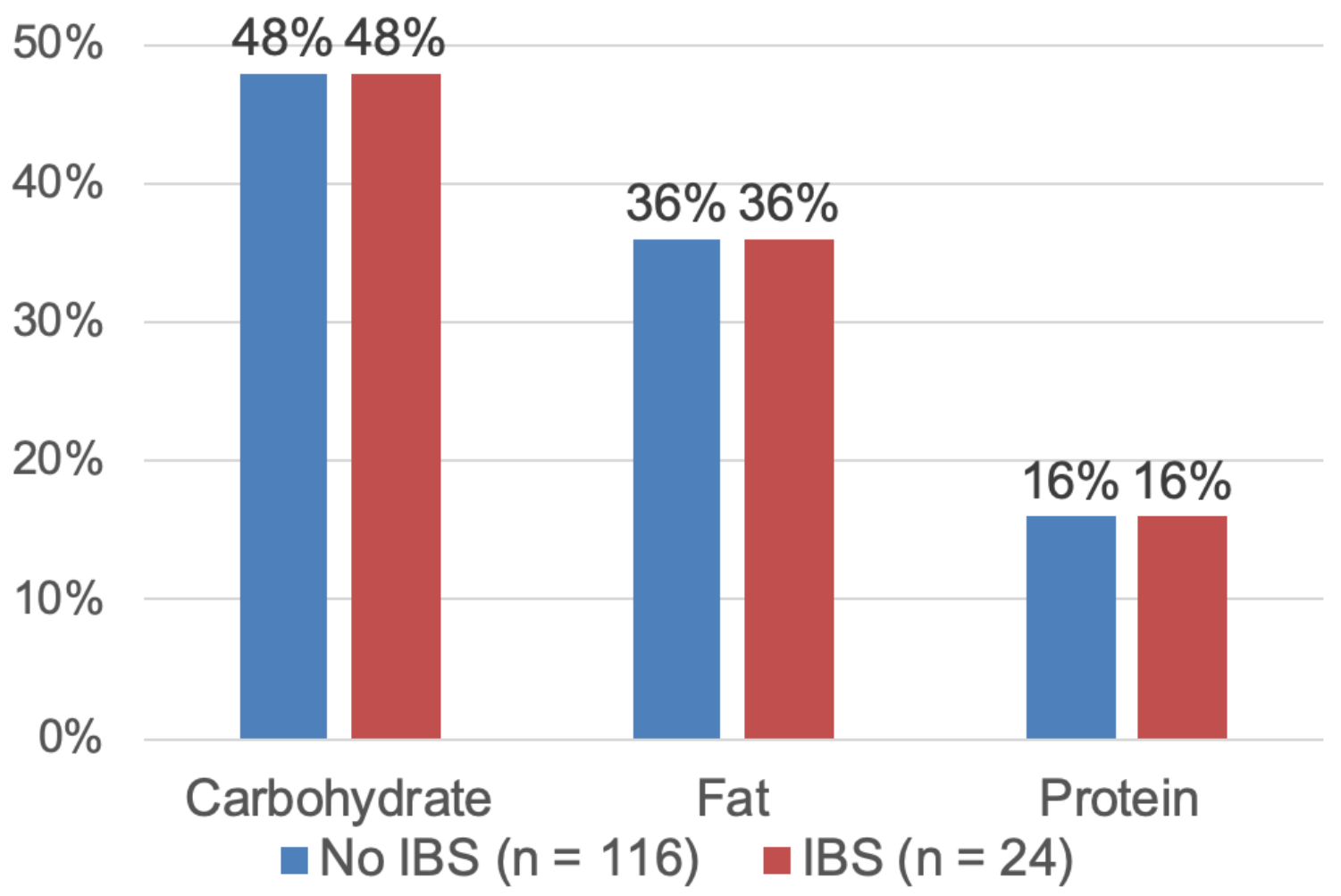


The average consumption of fructose, lactose, and polyols were collected from the FFQs and showed no difference in intake for any FODMAPSs in those with and without IBS (see Table 3).

Table 3. FODMAPs Intake as Measured by Vioscreen ${ }^{\mathrm{TM}}$ Report

\begin{tabular}{|c|c|c|c|c|c|c|}
\hline & \multicolumn{2}{|c|}{$\begin{array}{l}\text { Total sample } \\
(\mathrm{N}=140)\end{array}$} & \multicolumn{2}{|c|}{$\begin{array}{c}\text { No IBS } \\
(n=116)\end{array}$} & \multicolumn{2}{|c|}{$\begin{array}{c}\text { IBS } \\
(n=24)\end{array}$} \\
\hline & Median & $\begin{array}{l}\text { Mean } \pm \\
\text { SD }\end{array}$ & Median & $\begin{array}{l}\text { Mean } \pm \\
\text { SD }\end{array}$ & Median & $\begin{array}{l}\text { Mean } \pm \\
\text { SD }\end{array}$ \\
\hline Fructose $(\mathrm{g} / \mathrm{d})$ & 18.6 & $\begin{array}{l}27.0 \pm \\
35.0\end{array}$ & 16.9 & $25.1 \pm 34.1$ & 23.4 & $36.2 \pm 38.1$ \\
\hline Lactose $(\mathrm{g} / \mathrm{d})$ & 9.4 & $\begin{array}{l}12.6 \pm \\
13.0\end{array}$ & 8.5 & $12.4 \pm 13.5$ & 12.6 & $13.7 \pm 9.9$ \\
\hline $\begin{array}{l}\text { Sum polyols } \\
(\mathrm{g} / \mathrm{d})\end{array}$ & 0.7 & $0.8 \pm 0.6$ & 0.5 & $0.7 \pm 0.6$ & 0.8 & $0.9 \pm 0.5$ \\
\hline
\end{tabular}

Figure 2. Mean FODMAPs Intake in Participants with IBS and without IBS

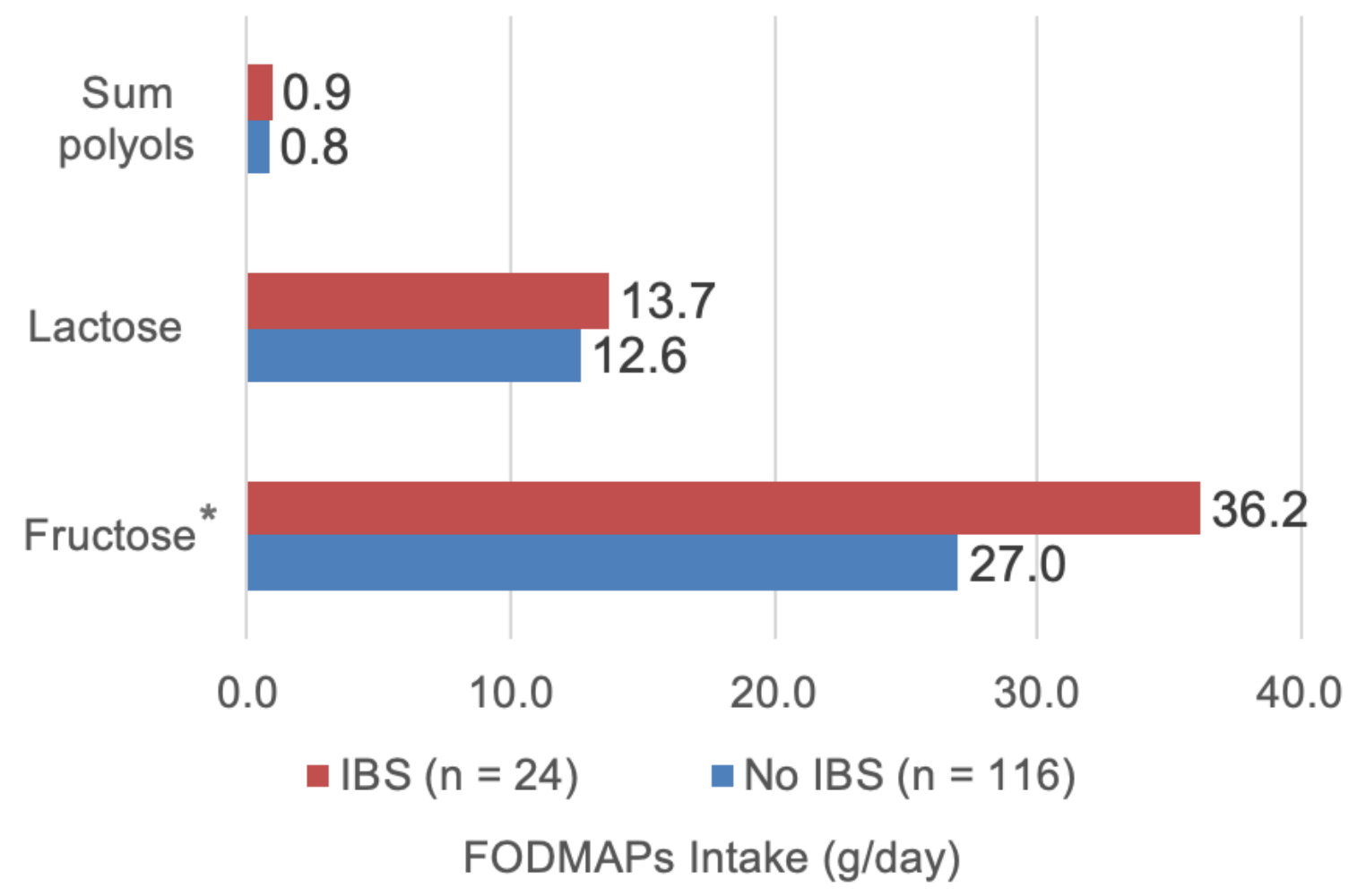

${ }^{*} \mathrm{P}$-value 0.156 
The average daily fiber intake is $21.3 \pm 15.4 \mathrm{~g} / \mathrm{d}$ in participants with IBS $(\mathrm{n}=24)$ (see Figure 3) and $18.6 \pm 10.3 \mathrm{~g} / \mathrm{d}$ in participants without IBS $(\mathrm{n}=116)$ (see Figure 3). Both two study groups' daily fiber consumption are higher than the average American fiber intake of 15$16 \mathrm{~g}^{17,22}$ (see Figure 3) and below the daily recommendation intake of $25-35 \mathrm{~g}^{17}$ (see Figure 3). It indicates that there is no significant difference for fiber intake between participants with IBS and without IBS.

Figure 3. Mean Fiber Intake per Day

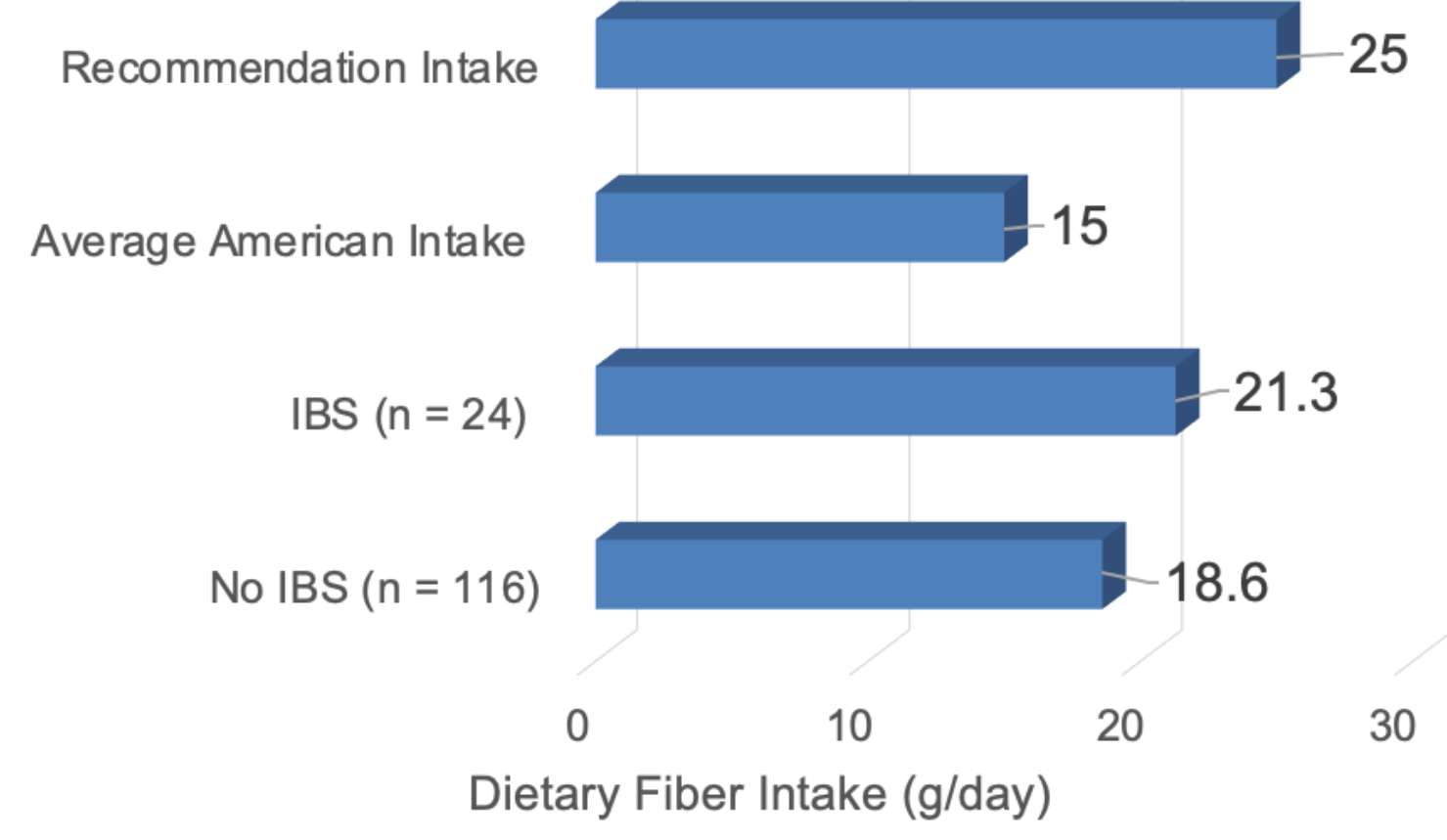

\section{Discussion}

To our knowledge, this is the first study to estimate FODMAP exposure using an FFQ in those with and without IBS to gain an understanding of usual dietary intake. Our population with IBS was slightly older (42 years of age) than other reports indicating that most patients with IBS report symptoms before age of 35 years. ${ }^{15}$ Based on the analysis of data that the National Health and Nutrition Examination Survey (NHANES) collected in 2003-2006, 32\% male and 38\% 
female population at age 31-50 years use MVMs. ${ }^{18}$ This was similar to our study which found nearly $40 \%$ of participants use MVMs. More than half of our cohort report little to no physical activity. A sedentary lifestyle has been associated with IBS with those engaging in $<1$ hour/wk having 1.27 times greater probability of IBS than those with $\geqslant 1$ hour $/ \mathrm{wk}^{21}$

For the RDN practicing in a GI clinic, it is important to understand if there are significant differences in the dietary patterns of those with and without IBS to improve counseling techniques and the delivery of dietary interventions. It is unknown if those patients with IBS are already self-restricting FODMAPs prior to education due to their ability to determine intolerance. It is also feasible that many patients seen in GI clinics self-restrict lactose and wheat due to perceived intolerance. It is well-documented that patients with IBS self-identify and restrict potential food triggers ${ }^{11}$. However, this was not apparent in this cohort as there were no differences between the kilocalorie, macronutrient, fiber and measured FODMAP intake between groups.

Dietary fiber intake is important to assess in this cohort since fiber is often adjusted my many patients and gastroenterologists within a GI clinic to impact bowel health. Average dietary fiber intake was higher than that of the typical American diet which is $15-16 \mathrm{~g} / \mathrm{day}$ for all individuals 2 years and older in 2009-2010 based on NHANES. ${ }^{22}$ However, FFQ data can overestimate energy intakes because of the length of the questionnaire and participants' abilities to estimate accurately the portion size of foods in the lists. ${ }^{23}$ Therefore, overestimated energy intake can lead the over-reported fiber intake as part of total energy intake. Additionally, people tend to over-report their intake of healthy foods (such as vegetables) and underreport unhealthy foods (such as candy) which can also impact fiber estimates. ${ }^{23}$ 
Specific FODMAP intake for the American population was unavailable for comparison; however, data reported in the literature allows us to have some discussion about comparisons of FODMAP intake in those with IBS. Specifically, our data indicates the average intake of fructose is quite variable at $36.2 \pm 38.1 \mathrm{~g} /$ day in those with IBS. The typical American diet contains fructose mainly as caloric sweeteners as high fructose corn syrup with the average intake of fructose increased from $37 \mathrm{~g} / \mathrm{d}$ to $49 \mathrm{~g} / \mathrm{d}$ from 1977 to $2005 .{ }^{16}$ Although our average fructose intake is less than that reported in the 2005 data, the dietary source is unknown. High fructose corn syrup is rapidly fermented by the GI tract and can lead to symptoms of IBS. Usual lactose consumption in Americans is unavailable; however, recommendations for dairy consumption for those following a $2000 \mathrm{kcal}$ diet is $2.5-3$ servings per day which is equivalent to $30-36 \mathrm{~g}$ of lactose per day since 1 cup milk contains $12 \mathrm{~g}$ lactose. ${ }^{24,25}$ However, based on the data collected by NHANES 2009-2010, the average Americans ages 2 and older take 1.9 servings of dairy products daily which equates to $22.8 \mathrm{~g}$ of lactose per day. ${ }^{26}$ This is not consistent with our cohort as our participants were consuming nearly $50 \%$ less than this estimate. Initial hypotheses would include that lactose intake is lower in a GI population compared to others given the spectrum of luminal diseases seen in clinic. Polyols are not considered an essential nutrient for inclusion in the American diet and usual intake in a US population is unknown. Reports in literature show that 10 to $20 \mathrm{~g}$ of sorbitol per day can lead to $90 \%$ and $100 \%$ of those in a healthy population to experience malabsorption, respectively. ${ }^{27}$ In patients with IBS, $5 \mathrm{~g}$ of sorbitol shows increased malabsorption. ${ }^{28}$ Our subjects consumed $0.8 \mathrm{~g} /$ day on average which is less than these reports indicated that perhaps this population is again already self-restricting these carbohydrates due to the impact on their GI symptoms. 
With average consumption of FODMAP intake identified, RDNs can understand the restrictive nature of a low FODMAP diet compared to habitual intake. These findings highlight that those in a GI clinic consume less lactose and polyols than the typical American population, indicated that there is already some restriction despite a diagnosis of IBS. Additionally, the use of the low FODMAP diet is being investigated in other GI conditions outside of IBS and perhaps these conditions are already self-restricting FODMAPs as well. Understanding dietary patterns in a GI population allows the RDN to target problem areas and reduce the time length of counseling on this complex dietary approach. In some clinics, the FFQ could be implemented as standard of care so that the RDN has this dietary pattern information available to assist with the consultation. In those clinics where this is not feasible, this research enhances the understanding of usual dietary patterns compared to other GI patients and supports that patients with IBS, although they may be self-restricting foods, they are not reducing overall FODMAP content of the diet. This should be replicated in other non-GI populations since food restrictions are not uncommon in many GI diagnoses. It could be hypothesized that overall FODMAP intake is lower in GI patients compared to healthy controls.

\section{Limitations}

Based on our knowledge, this is the first study to estimate FODMAP exposure using an FFQ in those with and without IBS to gain an understanding of usual dietary intake from a large sample size of 140 participants. However, the sample of those with IBS was small compared to our nonIBS group. Recruiting a larger sample of those with IBS might significantly change the averages reported here. It is unknown if these patients were already adjusting their dietary intake after an 
RDN consult which can potentially skew this data. Understanding changes in dietary patterns before and after an RDN consult would be beneficial for clarifying differences in these groups.

\section{Future Research}

Future research should focus on elucidating the food sources of FODMAPs in the dietary pattern of those with and without IBS. It is likely that we already restricting big classes of FODMAPs (ie., lactose and polyols) that hidden food sources could be related to continued symptoms identified in the IBS population.

\section{Conclusion}

Data states that high FODMAP foods are associated with the IBS gastrointestinal symptoms. In analyzing the dietary patterns of patients with and without IBS, this study found that patients with IBS do not consume significantly less fructose, lactose, and polyols compared to patients without IBS. However, the total population appears to consume much less than what is reported in NHANES data. RDNs should evaluate the dietary patterns before the education of low-FODMAPs to ensure the education is targeting patient-specific high FODMAPs foods or potential trigger foods. 


\section{Reference}

1. Nutrition Care Manual. Conditions. Nutritioncaremanual.org. https://www.nutritioncaremanual.org/topic.cfm?ncm_category_id=1\&lv1 $=5522 \& 1 \mathrm{v} 2=19$ $589 \&$ ncm_toc_id=19589\&ncm_heading $=$ Nutrition\%20Care\#

2. Nahikian- $-\bar{N}$ elms M. Nutrition Therapy and Pathophysiology. Fourth ed. Boston, MA: Cengage; 2020.

3. Endo Y, Shoji T, Fukudo S. Epidemiology of irritable bowel syndrome. Ann Gastroenterol. 2015;28(2):158-159.

4. El-Salhy M. Recent developments in the pathophysiology of irritable bowel syndrome. World J Gastroenterol. 2015;21(25):7621-7636. doi:10.3748/wjg.v21.i25.7621

5. VioScreen FFQ. Available online: https://www.viocare.com/vioscreen.html.

6. Saha L. Irritable bowel syndrome: pathogenesis, diagnosis, treatment, and evidencebased medicine. World J Gastroenterol. 2014;20(22):6759-6773. doi:10.3748/wjg.v20.i22.6759

7. Irritable Bowel Syndrome. American College of Gastroenterology. https://gi.org/topics/irritable-bowel-syndrome/.

8. Chey WD, Kurlander J, Eswaran S. Irritable Bowel Syndrome: A Clinical Review. JAMA. 2015;313(9):949-958. doi:10.1001/jama.2015.0954

9. Schmulson MJ, Drossman DA. What Is New in Rome IV. J Neurogastroenterol Motil. 2017;23(2):151-163. doi:10.5056/jnm16214

10. Mansueto, P., Seidita, A., D'Alcamo, A. and Carroccio, A. (2015), Role of FODMAPs in Patients With Irritable Bowel Syndrome. Nutrition in Clinical Practice, 30: 665-682. doi:10.1177/0884533615569886

11. Böhn L, Störsrud S, Liljebo T, et al. Diet Low in FODMAPs Reduces Symptoms of Irritable Bowel Syndrome as Well as Traditional Dietary Advice: A Randomized Controlled Trial. Gastroenterology. 2015; 149:1399-1407.

12. Werlang ME, Palmer WC, Lacy BE. Irritable Bowel Syndrome and Dietary Interventions. Gastroenterol Hepatol (N Y). 2019;15(1):16-26.

13. Böhn L, Störsrud S, Törnblom H, Bengtsson U, Simrén M. Self-reported food- related gastrointestinal symptoms in IBS are common and associated with more severe symptoms and reduced quality of life. Am J Gastroenterol. 2013;108(5):634- 641.

14. Hustoft TN, Hausken T, Ystad SO, et al. Effects of varying dietary content of fermentable short-chain carbohydrates on symptoms, fecal microenvironment, and cytokine profiles in patients with irritable bowel syndrome. Neurogastroenterol Motil. 2017;29(4).

15. Canavan C, West J, Card T. The epidemiology of irritable bowel syndrome. Clin Epidemiol. 2014;6:71-80. Published 2014 Feb 4. doi:10.2147/CLEP.S40245

16. Zheng Z, Harman JL, Coresh J, et al. The Dietary Fructose:Vitamin C Intake Ratio Is Associated with Hyperuricemia in African-American Adults. J Nutr. 2018;148(3):419426. doi:10.1093/jn/nxx054

17. UCSF Health. Increasing Fiber Intake. ucsfhealth.org. https://www.ucsfhealth.org/education/increasing-fiber-intake. Published October 31, 2019. 
18. Office of Dietary Supplements - Multivitamin/mineral Supplements. NIH Office of Dietary Supplements. https://ods.od.nih.gov/factsheets/MVMS-HealthProfessional/.

19. Khayyat Y, Attar S. Vitamin D Deficiency in Patients with Irritable Bowel Syndrome: Does it Exist?. Oman Med J. 2015;30(2):115-118. doi:10.5001/omj.2015.25

20. Vitamin D supplements could ease painful IBS symptoms. ScienceDaily. https://www.sciencedaily.com/releases/2018/01/180125110806.htm. Published January 25, 2018.

21. Sadeghian M, Sadeghi O, Hassanzadeh Keshteli A, Daghaghzadeh H, Esmaillzadeh A, Adibi P (2018) Physical activity in relation to irritable bowel syndrome among Iranian adults. PLoS ONE 13(10): e0205806. https://doi.org/10.1371/journal.pone.0205806

22. U.S. Department of Agriculture, Agricultural Research Service. 2013. What We Eat in America Food Categories 2009-2010. Available: www.ars.usda.gov/ba/bhnrc/fsrg . Accessed 10/29/13

23. Institute of Medicine (US) Committee on Dietary Risk Assessment in the WIC Program. Dietary Risk Assessment in the WIC Program. Washington (DC): National Academies Press (US); 2002. 5, Food-Based Assessment of Dietary Intake. Available from: https://www.ncbi.nlm.nih.gov/books/NBK220560/

24. Quann EE, Fulgoni VL 3rd, Auestad N. Consuming the daily recommended amounts of dairy products would reduce the prevalence of inadequate micronutrient intakes in the United States: diet modeling study based on NHANES 2007-2010. Nutr J. 2015;14:90. Published 2015 Sep 4. doi:10.1186/s12937-015-0057-5

25. Suchy FJ et al. NIH Consensus Development Conference Statement: lactose intolerance and health. NIH Consens State Sci Statements 2010;27:1-27.

26. U. S. Department of Agricultural Service. 2013. Food Patterns Equivalents Intakes from Food: Mean Amounts Consumed per Individual, by Gender and Age, What We Eat In America, NHANES 2009-2010. Available at: www.ars.usda.gov/ba/bhnrc/fsrg.

27. Corazza GR, Strocchi A, Rossi R, Sirola D, Gasbarrini G. Sorbitol malabsorption in normal volunteers and in patients with coeliac disease. Gut 1988;29:44-8.

28. Fernández-Bañares F, Esteve-Pardo M, de Leon R, Humbert P, Cabré E, Llovet JM, Gassull MA Am J Gastroenterol. 1993 Dec; 88(12):2044-50. 